\title{
Editorial: Theoretical Approaches to Community Ecology
}

\author{
Luís Borda-de-Água ${ }^{1,2,3 *}$, Paulo A. V. Borges ${ }^{4}$ and John M. Halley ${ }^{5}$ \\ ${ }^{1}$ Centro de Investigação em Biodiversidade e Recursos Genéticos (CIBIO), InBIO Laboratório Associado, Universidade do \\ Porto, Vairão, Portugal, ${ }^{2}$ Centro de Investigação em Biodiversidade e Recursos Genéticos (CIBIO), InBIO Laboratório \\ Associado, Instituto Superior de Agronomia, Universidade de Lisboa, Lisboa, Portugal, ${ }^{3}$ BIOPOLIS Program in Genomics, \\ Biodiversity and Land Planning, Centro de Investigação em Biodiversidade e Recursos Genéticos (CIBIO), Vairão, Portugal, \\ ${ }^{4} \mathrm{CE} 3 \mathrm{C}$-Centre for Ecology, Evolution and Environmental Changes/Azorean Biodiversity Group, Faculty of Agriculture and \\ Environment, Department of Environmental Sciences and Engineering, Universidade dos Açores, Angra Do Heroísmo, \\ Portugal, ${ }^{5}$ BET-EcoLab, Department of Biological Applications and Technology, University of loannina, loannina, Greece
}

Keywords: analytical methods, biodiversity, community ecology, patterns and processes, theoretical ecology

\section{Editorial on the Research Topic}

\section{Theoretical Approaches to Community Ecology}

Our understanding of the processes determining community diversity and how to interpret community patterns is a work in progress. Since the landmark works of MacArthur and Wilson $(1963,1967)$ on Island Biogeography nearly 60 years ago, we have seen many exciting theoretical advances in community ecology. But is it enough? The last two decades have seen remarkable growth in the availability of data, especially biodiversity data, via satellite technology, next-generation sequencing and an explosion of open access online databases. At the same time, the expanding rate and complexity of key drivers of biodiversity loss, such as habitat fragmentation, invasive species and climatic change brings unprecedented threats to ecological communities (Borges et al., 2019). The conjunction of these changes is creating both new problems and new applications for Community Ecology. Due to this combination of recent challenges and opportunities, this is an especially appropriate moment to consider the role of theory in the development of new approaches to Community Ecology, of biodiversity patterns and the processes that influence them.

Theory is an important part of Community Ecology. Ecological communities are so complex that even a small part of any of them presents a world in itself, with diversity and complexity beyond the scope of any model to describe fully (Cardoso et al. in this special issue). So, every community theory is incomplete (Halley and Pimm in this special issue). Nevertheless, even crude theories provide essential guidance towards fuller understanding. Theory with a strong mathematical basis is a characteristic of any mature science. It describes in a mathematical way the first principles and mechanisms by which processes operate and interact, and this enables the science to renew itself when exposed to new types of data or when confronted with new types of problems. Such theory is thus necessary for ecology in general, and for Community Ecology in particular, because it enables Community Ecology to develop as a predictive science. In this way, Community Ecology can itself evolve and provide tools and perspectives that will assist our societies to respond to current and future environmental challenges.

This Research Topic explores the state-of-the-art in theoretical Community Ecology and reveals significant new research on the patterns and processes that drive communities. For this issue, we brought together researchers from ecology but also researchers with different backgrounds with common interests in Ecology, such as Physics, Mathematics and Computer Science. Thus, these articles present insights into Community Ecology from various perspectives, with different theoretical and methodological approaches, covering studies on communities at all scales, from 
individuals to ecosystems, as well as multi-scale approaches. The papers were organised into five different categories: "community modelling," "patterns of species abundance and rarity," "landuse change and habitat fragmentation," "modelling multi-species dynamics," and "speciation and evolution."

The first two articles in the section Community modelling are clearly methodological. Cardoso et al. brings a novel approach using artificial intelligence and machine learning to detect patterns and predict outcomes in ways that often resemble human reasoning. They used a particular method called symbolic regression that allows the evolution of humaninterpretable formulas to explain natural laws. For example, Cardoso et al. were able to find a new general model that explains why some islands have more species than others, being able to simulate the GDM model (Whittaker et al., 2008). This publication shows that evolving free-form equations purely from data, often without prior human inference or hypotheses, may represent a powerful tool in the arsenal of a discipline as complex as ecology. These authors also applied their approach successfully to other models in community ecology, testing the robustness of symbolic regression at extreme levels of noise when searching for the species-area relationship and finding new formulas for species richness estimators. The next methodological advance comes from the manuscript of Carvalho and Cardoso in which a new framework is proposed to decompose overall differentiation among hypervolumes into two distinct components: replacement of space (niche shifts) and net differences between space amplitudes (niche contraction/expansion processes). The authors demonstrate that it is possible to disentangle different processes underlying niche partitioning between coexisting species, offering novel insights to understand the drivers of niche partitioning and allowing the address of new niche- and trait-based questions in community ecology.

Eldijk et al. propose a simple eco-evolutionary model, the Community-Wide Rescue (CWR) model, in which when a community faces environmental deterioration, each species within the community is forced to undergo adaptation or become extinct. The authors show that when implementing their neutral individual based simulation model rare species become extinct very rapidly, which raises concerns with the ongoing global changes, as many ecosystems face irreversible human induced environmental change.

Finally in this topic, Halley and Pimm developed the niche-hypervolume concept of the community into a powerful model of community dynamics. The authors lay out a framework to answer similar questions to those addressed by Hubbell's neutral theory, but with a niche-based perspective and explore the behaviour using both mathematical analysis and computer simulations. This dynamic model reproduces key patterns of communities, such as lognormal species abundance distributions, lognormality of populations in the time domain, $1 / f$-noise population fluctuations, extinction debt and logarithmic species-time curves. It also provides a powerful framework to explore significant ideas in ecology, such as community turnover and the drift of ecological communities into evolutionary time.
Several articles in the section Patterns of Species abundance and rarity used the species abundance distribution (SAD) as their main tool to describe and understand biodiversity patterns. For instance, the main objective of Tsafack et al. was to assess the influence of environmental conditions on the SADs of carabid beetles. They studied communities from three grassland ecosystems (desert, typical and meadow steppes) in China, and fitted the SADs using a variety of models: the meta-community zero sum multinomial, the lognormal, Fisher's logseries, and unimodal and multimodal gambin distributions. Importantly, they found that the parameters of the distributions were a function of the scale of analysis. Overall, the communities at different scales were equally well fitted by the meta-community zero-sum multinomial and Fisher's logseries.

Also realising the importance of looking at communities at different scales, Alirezazadeh et al. analysed the functional diversity of arthropod communities in the Azores. The main argument for using functional diversity was that some species play similar roles and therefore grouping species according to their characteristics may be more relevant to understanding community dynamics. However, the authors moved away from the typical approach of looking at only one scale and focused on characterising patterns of functional diversity across spatial scales, similar to what is routinely done with taxonomic diversity, such as the species-area relationship (SAR). In addition to studying the equivalent of SAR to functional groups, Alirezazadeh et al. applied, and further developed, methods previously introduced by some of the authors to study the scaling properties of SADs (e.g., Borda-de-Água et al., 2012). They found that scales of functional diversity are quantitatively different from taxonomic diversity and discuss the implications of this finding if the dynamics of communities are driven primarily by the characteristics of their functional diversity.

The importance of studying the shape of the SADs is also emphasised by Antão et al. These authors examined 11 largescale datasets of different taxa and compared the fit provided by different models at different scales. They used the logseries, the lognormal and a multimodal distribution. A major result of this work is that multimodality is present for a wide range of scales, and concluded that two of the major theories, namely the Neutral Theory of Biodiversity and Biogeography (Hubbell, 2001) and the Maximum Entropy Theory (Harte, 2011) of ecology are unable to justify the variability of SAD shapes observed. The authors conclude that critical developments are still required in order to fully understand the shapes of the SADs.

Finally in this section, Brush and Harte emphasise the importance of relating observed patterns with the underlying mechanisms and processes. To this end, the authors developed a model that integrates pattern, by spatially distributing individuals of a species based on the rules of the Maximum Entropy Theory of Ecology (Harte, 2011), and mechanism, through the relationship between the probability of an individual to die and the abundance of its species. In order to compare the results of the model with real data, they used two data sets, one with information on the abundance of tropical tree and shrub species in a 50 ha plot in Barro Colorado Island and another with information on plant abundance on $64 \mathrm{~m}^{2}$ serpentine grassland 
plot. The authors conclude that their model predicts that an increase in intraspecific negative density dependence is related to more random spatial patterning of species spatial distributions.

One key driver of the erosion of biodiversity is habitat fragmentation and degradation (Borges et al., 2019). Three articles in this special issue, in the section Land-use change and habitat fragmentation, deal with this important theme. Staude et al. used a space-for-time substitution approach, surveying bird communities in native grassland sites in 31 regions with differing levels of habitat conversion. Their results support predictions of the theory of the ideal free distribution, and suggests that native habitat remnants are very important temporary refugia for specialist bird species. The results obtained can partly explain the segregation of habitat specialist and generalist birds observed in many agricultural landscapes, in which higher abundance of specialists are found in native grassland patches.

Changing from empirical to a modelling approach Chetcuti et al. used a multi-landcover, landscape-scale, individual-based model to show that the gamma diversity of species for whom the focal land cover is a suitable habitat, declined under fragmentation per se. Interestingly, specialists were unaffected by fragmentation per se. These authors also found that that betadiversity and gamma-diversity increased overall even without differences among species in habitat specialisations. The findings of Chetcuti et al. will help to inform the fragmentation per se debate, showing that effects on biodiversity can be negative or positive, depending on species' competitive abilities and dependency on the fragmented land cover.

Finally, Wayman et al. used a generalised dissimilarity modelling approach to demonstrate that there is moderate and unique proportion of the variance explained by geographical distance per se, which could highlight the role of dispersal limitation in community dissimilarity. Moreover, they observed a key role for environmental filtering both for taxonomic nestedness and functional nestedness, with important conservation implications in the face of a warming climate and future land use change.

Communities of organisms are made up of populations of individual species. Thus, all community dynamics ultimately rest on the dynamics of populations. For example, the rise and fall of species richness in a community may be seen as the number of species in a community having numbers greater than some threshold. This special issue has three such papers, in the section Modelling multi-species dynamics.

Seto and Iwasa explored how thermodynamics illuminates population interactions in microbial communities. Many traditional model populations, especially for microbial systems, can be divided into producers and consumers. However, most investigations are focused on systems based around solar energy, while many communities are based around other forms of energy, such as hot springs, hydrothermal vent systems or even gut microbes. Does it really matter, which energy is the basis of a community? Seto and Iwasa argue that it does and they introduce theoretical approaches based on differential equations that incorporate thermodynamics to highlight characteristic interactions between species in the microbial community. In contrast to light-dependent producers, which compete with one another for light, producers using Gibbs free energy (chemical energy) can have cooperative interactions with each other through the effects of the relative quantities of products and reactants on the available chemical energy. This is an exciting insight into how communities work at the microbial level with non-solar inputs.

Akimenko also used a resource-consumer model for interacting populations. However, in this case, the system of coupled differential equations was generalised in the direction of seeing what are the effects of age-structure and delays in the basic equations. The model of consumer population dynamics was described by a delayed transport equation, and the dynamics of resource patches are described by ODEs with saturated intake rate. The delay described the digestion period of generalist consumers and is included in the calorie intake rate, which impacts the consumer's fertility and mortality and affects what happens if the behaviour of the food resource species changes when consumer population grows. Usually, the incorporation of delays into systems of consumer-resource equations, causes increasing instability. However, here the delay caused by the digestion period of a generalist consumer did not cause local asymptotical instabilities of consumer population at the semi-trivial and non-trivial equilibria. These theoretical results (supported by simulations) also have applications to metapopulation dynamics as well as to multispecies models.

$\mathrm{He}$ et al. again focus on a microbial system but this time it is one based on parasitism. Their study of frequency-dependent competition between strains of Plasmodium falciparum also belongs in the fields of evolution and the epidemiology of malaria. The focus here is on how populations of P. falciparum exhibit a vast diversity of the var gene encoding its major surface antigen, with each parasite comprising multiple copies from this diverse gene pool. The authors used a combination of stochastic agent-based models, principal component analysis and network analyses to test various theoretical results, where assembly of local parasite diversity occurs under frequency-dependent selection.

Both papers in the section Speciation and evolution focus on speciation. Interestingly, both concluded that dispersal ability plays a major role in determining the characteristics of the observed phylogenetic trees, their regions of clustering, and the associated speciation rates.

Tao et al. address the question of why some lineages have higher speciation rates than others when colonising a given region. In order to study the radiation of different lineages the authors developed a spatially explicit neutral model with protracted speciation; importantly, this model was free from the complexity of explicit niches. The main parameters controlling the radiation sizes were the geographic isolation and dispersal ability. The authors found an intermediate dispersal value where lineages may differ considerable in radiation size: if for stochastic reasons dispersal is very low, this leads to rapid speciation events that were named a "radiation cascade." Tao et al. suggest that such radiation events may be common, and not necessarily only a feature of neutral models, and they may occur without the need to invoke niche differentiation.

Also using a theoretical model, Kallimanis et al. studied whether metrics of phylogenetic clustering are able to detect 
a taxon speciation history. In this study, the feature that led to important outcomes was dispersal. When dispersal was not present, then only when the incipient species was present in the speciation region could some metrics identify regions of high speciation. On the other hand, when dispersal was present and speciation occurred in a spatially continuous way, then there was a connexion between the speciation rate and the clustering of the phylogenetic tree; if speciation happened randomly in space, then such a relationship was not observed. The authors conclude that phylogenetic clustering can only be an indicator of speciation under a limited set of conditions.

Altogether, the articles in this special issue show that theory community ecology is a vibrant area of research, employing a wide variety of approaches. It is important that continuing theoretical efforts in these directions take advantage of concurrent and increasingly rapid progress in data collection

\section{REFERENCES}

Borda-de-Água, L., Borges, P. A. V., Hubbell, S. P., and Pereira, H. M. (2012). Spatial scaling of species abundance distributions. Ecography $\quad 35, \quad 549-556 . \quad$ doi: $\quad 10.1111 /$ j.1600-0587.2011.07 128.x

Borges, P. A. V., Gabriel, R., and Fattorini, S. (2019). "Biodiversity erosion: causes and consequences," in Life on Land. Encyclopedia of the UN Sustainable Development Goals, eds W. Leal Filho, A. Azul, L. Brandli, P. Özuyar, and T. Wall (Cham: The Springer Nature), $1-10$.

Harte, J. (2011). Maximum Entropy and Ecology: A Theory of Abundance, Distribution, and Energetics. Oxford: Oxford University Press.

Hubbell, S. P. (2001). The Unified Neutral Theory of Biodiversity and Biogeography (MPB-32). Princeton, NJ: Princeton University Press.

MacArthur, R. H., and Wilson, E. O. (1963). An equilibrium theory of insular zoogeography. Evolution 17, 373-387. doi: 10.1111/j.1558-5646.1963.tb03 295.x

MacArthur, R. H., and Wilson, E. O. (1967). The Theory of Island Biogeography. Princeton, NJ: Princeton University of data. We believe that the papers of this special issue will foster such progress and stimulate further advances in theoretical approaches in community ecology and collaboration among researchers using different methodological approaches.

\section{AUTHOR CONTRIBUTIONS}

All authors have made substantial contributions to the Editorial and in organising and editing the Research Topic.

\section{FUNDING}

LB-d-A and PAVB were financed through Portuguese national funds through FCT-Fundação para a Ciência e a Tecnologia, I.P. (LB-d-A: under the Norma Transitória-DL57/ 2016/CP1440/CT0022; PAVB: UIDB/00329/2020-2024).

Whittaker, R. J., Triantis, K. A., and Ladle, R. J. (2008). A general dynamic theory of oceanic island biogeography. J. Biogeogr. 35, 977-994. doi: $10.1111 /$ j.1365-2699.2008.01892.x

Conflict of Interest: The authors declare that the research was conducted in the absence of any commercial or financial relationships that could be construed as a potential conflict of interest.

Publisher's Note: All claims expressed in this article are solely those of the authors and do not necessarily represent those of their affiliated organizations, or those of the publisher, the editors and the reviewers. Any product that may be evaluated in this article, or claim that may be made by its manufacturer, is not guaranteed or endorsed by the publisher.

Copyright (C) 2022 Borda-de-Água, Borges and Halley. This is an open-access article distributed under the terms of the Creative Commons Attribution License (CC BY). The use, distribution or reproduction in other forums is permitted, provided the original author(s) and the copyright owner(s) are credited and that the original publication in this journal is cited, in accordance with accepted academic practice. No use, distribution or reproduction is permitted which does not comply with these terms. 